\title{
Two Approaches to the Study of a Controversial Relationship: Cutaneous Photosensitivity and Anti-Ro/SS-A Autoantibodies
}

\author{
Mariela L. Paz, Eliana M. Cela, Alejandro Ferrari, Adrián D. Friedrich, Juliana Leoni, \\ Daniel H. González Maglio
}

Immunology Department, Pharmacy and Biochemistry School, University of Buenos Aires, Buenos Aires, Argentina.

Email: mlpaz@ffyb.uba.ar

Received November $5^{\text {th }}, 2013$; revised December $5^{\text {th }}, 2013$; accepted December $12^{\text {th }}, 2013$

Copyright (c) 2014 Mariela L. Paz et al. This is an open access article distributed under the Creative Commons Attribution License, which permits unrestricted use, distribution, and reproduction in any medium, provided the original work is properly cited. In accordance of the Creative Commons Attribution License all Copyrights @ 2014 are reserved for SCIRP and the owner of the intellectual property Mariela L. Paz et al. All Copyright (C) 2014 are guarded by law and by SCIRP as a guardian.

\section{ABSTRACT}

Background: Autoantibodies (Aabs) are the hallmark of numerous systemic autoimmune pathologies (SAPs), for instance anti-Ro/SS-A Aabs are usually found in Systemic Lupus Erythematosus (SLE) and Sjögren's Syndrome. Cutaneous photosensitivity (CP) is found in most forms and subsets of LE and consists of a skin rash as a result of unusual reaction to sunlight. There are many theories which relate specifically the presence of circulating antiRo/SS-A Aabs with the CP phenomenon, though there are several studies which are in disagreement. Results: In this study we analyzed the relationship between $\mathrm{CP}$ and anti-Ro Aabs by means of two approaches. The first one included an in vitro model where we evaluated by flow cytometry the binding capacity of affinity-purified Aabs to autoantigens relocalized on apoptotic keratinocyte's surface. We found that there was no relationship between the binding capacity of serum from 10 selected patients or their corresponding purified anti-Ro52 and anti-Ro60 Aabs, and the presence or absence of CP, neither with the SAPs. The in vivo model consisted of Hairless SKH:1 mice which were induced to produce anti-murine Ro52 and/or Ro60 Aabs and were subsequently irradiated with UVB light. We evaluated the skin histology and also the epidermal production of TNF- $\alpha$. We found no differences between the groups in neither of the parameters evaluated. Conclusions: These results agree with some studies on the role of the Aabs in CP, considering anti-Ro Aabs not as the only responsible for the manifestation; and disagree with many other authors, who believe in the strong association between these two events.

\section{KEYWORDS}

Cutaneous Photosensitivity; Purified Anti-Ro/SS-A Autoantibodies; SKH-1 Hairless Mice; UVB Irradiation; TNF- $\alpha$

\section{Introduction}

Exposure to sunlight has long been associated with exacerbation of Systemic Lupus Erythematosus (SLE), not only of cutaneous manifestations like photosensitivity, but also of several disease symptoms such as weakness, fatigue and joint pain [1]. Up to $90 \%$ of patients with SLE report cutaneous photosensitivity (CP), which-according to the American College of Rheumatologyconsists of a skin rash as a result of unusual reaction to sunlight, by patient history or physician observation [2-3].
$\mathrm{CP}$ is found in most forms and subsets of Lupus Erythematosus (LE) like Subacute Cutaneous (SCLE), Neonatal (NLE), Discoid (DLE) and SLE, and also in patients with Dermatomyositis [4-6]. The main responsible for the harmful effects of sunlight is ultraviolet radiation (UVr) - specially UVB (280 - $320 \mathrm{~nm})$, though it has also been demonstrated that UVA (320 - $400 \mathrm{~nm}$ ) can contribute to the induction of LE skin lesions [7].

Autoantibodies (Aabs) are the hallmark of numerous systemic autoimmune pathologies (SAPs). These Aabs 
have many nuclear targets, like proteins (Sm, histones, etc.) DNA and ribonucleoprotein complexes (RNPs) such as the RoRNP, among others. This complex is composed by a RNA molecule and three proteins: La/SS-B of 47 $\mathrm{kDa}$ located in the nucleus, Ro/SS-A of $52 \mathrm{kDa}$ and Ro/SS-A of $60 \mathrm{kDa}$, which can be found in the nucleus and also free in the cytoplasm [8]. The incidence of anti-Ro/SS-A Aabs is different according to the SAP involved: in primary Sjögren's Syndrome (pSS) and secondary SS (sSS) is about $80 \%-95 \%$ and in SLE these Aabs appear in 30\% - 60\% of all cases [9-11]. Although anti-Ro/SS-A $52 \mathrm{kDa}$ and anti-Ro/SS-A $60 \mathrm{kDa}$ Aabs are usually evaluated together as anti-Ro/SS-A, it is useful to detect them separately because each of them have different connotations. For instance, it has been proven that anti-Ro/SS-A $52 \mathrm{kDa}$ Aabs are responsible for the pathogenesis of congenital heart block [12] and it has been reported that anti-Ro/SS-A $60 \mathrm{kDa}$ Aabs are associated with glandular dysfunction in SS patients [13]. Additionally, anti-Ro/SS-A $60 \mathrm{kDa}$ Aabs have recently been identified as an early marker which can occur years before SLE disease onset and have been proposed as an initiating target that promotes epitope spreading to additional autoantigenic specificities [14].

Apoptosis is an active process that leads to the ordered removal of living cells avoiding the generation of an inflammatory environment. This is desirable in most of the cases, though in SAPs this might not be so favorable. It has been demonstrated that UVr driven apoptotic keratinocytes relocate RNPs towards the cell membrane $[15,16]$ and this event could, on a first instance, induce the production of Aabs [17]. Subsequently these circulating Aabs could bind to the apoptotic keratinocytes' membrane, producing Aabs mediated cytotoxicity, finally leading to an unwanted inflammatory response, which under normal conditions would not take place [18]. These events are proposed to be relevant in the generation of cutaneous damage in SAPs patients.

It stands to reason why anti-Ro/SS-A Aabs have traditionally been associated with the CP phenomenon [1921]. Nevertheless, there are some facts which are in disagreement with the previous statement. To begin with, there are epidemiological data which indicate that there is not a significant direct association between anti-Ro/ SS-A Aabs and CP [22-24]; moreover there is the fact that $75 \%$ of pSS's patients do have anti-Ro/SS-A Aabs, many with high Aabs titers, but not CP [25,26]; furthermore there is the occurrence of $\mathrm{CP}$ in individuals that do not show any kind of disease, neither any circulating Aabs [27]. Anyhow, beyond all this controversy the actual mechanism which triggers CP has not been fully elucidated yet, though the relationship between $\mathrm{CP}$ and anti-Ro/SS-A Aabs has been studied focusing on differ- ent aspects. These include the expression of autoantigens in the skin [28], the presence of certain Aabs [21] and also the relation with apoptotic cell death induced by UVr [29], but the study of the above mentioned association using purified anti-Ro/SS-A Aabs has been poorly explored.

The study of this association in an animal model is also vacant, though some approaches have been made, such as the study of the deposition of immunoglobulins in human skin-grafted mice [30] and the induction of erythema in animals with passive transferred Aabs [31] However, these studies do not include the analysis of skin inflammatory mediators-such as TNF- $\alpha$-which has been related to the pathogenesis of photosensitive lupus. This relation is based on studies of human TNF- $\alpha$ polymorphisms in lupus patients and measures of their cytokine serum levels [32] Nonetheless, there has been contradictory data about this matter, since TNF- $\alpha$ blocking agents are able to induce lupus-like syndromes, including skin disease $[4,33]$.

In this study we analyzed the relationship between CP and anti-Ro/SS-A Aabs by means of two approaches. The first one includes an in vitro model where we evaluated the binding capacity of purified Aabs to apoptotic keratinocyte's cell surface by flow cytometry. We selected 10 patients from a previous larger study, according to their clinical characteristics and laboratory findings, and affinity-purified anti-Ro/SS-A of $52 \mathrm{kDa}$ and antiRo/SS-A of $60 \mathrm{kDa}$ Aabs from their serum samples. We analyzed the existence of a relationship between the binding profile to apoptotic keratinocytes of the serum and pure Aabs of each patient, and the presence or absence of CP. The in vivo model consists of Hairless SKH:1 mice which were induced to produce anti-murine Ro/SS-A 52 and/or Ro/SS-A 60 Aabs and were subsequently irradiated with UVB light. We evaluated the skin histology and also the epidermal production of TNF- $\alpha$.

\section{Materials and Methods}

\subsection{Ethics Statement}

Serum samples from patients and healthy volunteers were obtained from the Rheumatology Service of the Hospital de Clínicas José de San Martín, Buenos Aires, Argentina. All studies were conducted, and all samples were obtained with written, informed consent under Institutional Review Board on Human Ethics approved protocols from the mentioned Hospital. The study has been performed in accordance with the ethical standards laid down in the 1964 World Medical Association Declaration of Helsinki.

Animals were used in compliance with the research animal use guidelines established by the Consejo $\mathrm{Na}$ - 
cional de Investigaciones Científicas y Técnicas (CONICET-Argentina) and with the EC Directive 86/609/EEC [34]. The protocol was approved by the Institutional Review Board on Ethics of Animal Experiments of the Instituto de Estudios de la Inmunidad Humoral (IDEHU) from CONICET-University of Buenos Aires.

\subsection{Patients, Samples and Controls}

A cohort of 169 individuals were examined at the Rheumatology Service of the Hospital de Clínicas José de San Martín, as part of a previous larger study [24], then 10 of those patients were selected for this study, according to their clinical characteristics and laboratory findings. Diagnoses of SLE, SS, Rheumatoid Arthritis (RA) and CP were made according to the criteria adopted in the abovementioned previous study.

Twenty milliliters (ml) of serum were obtained from each of the 10 patients and stored at $-20^{\circ} \mathrm{C}$ until the purification processes began. Sera from 10 healthy subjects (normal human serum, NHS) were used as control; $1 \mathrm{ml}$ of each one was pooled and stored at $-20^{\circ} \mathrm{C}$. All $10 \mathrm{NHS}$ obtained were tested individually for ANA, anti-Ro/SS-A 52 and anti-Ro/SS-A $60 \mathrm{kDa}$, being the results always negative. Immunoglobulin G (IgG) concentration was measured in sera from the 10 patients and also in the NHS pool by radial immunodiffusion (Diffu-Plate, Biocientífica, Argentina).

\subsection{Affinity Purification of Aabs against Recombinant Ro/SS-A 52 kDa and Ro/SS-A 60}

Recombinant human Ro/SS-A $52 \mathrm{kDa}$ and Ro/SS-A 60 $\mathrm{kDa}$ (Ro52 and Ro60 hereafter) were cloned and expressed in an E-coli system, where a N-terminal 6× Histidine-tag was added to the proteins and then they were purified using an immobilized metal ion adsorption chromatography, as described before [24]. Aabs against either Ro52 or Ro60 proteins were isolated from sera by affinity chromatography, using the corresponding recombinant protein coupled to CNBr-activated Sepharose 4B (GE Healthcare, Little Chalfont, Buckinghamshire, United Kingdom) according to the manufacturer's instructions. Briefly, $1 \mathrm{ml}$ of $\mathrm{CNBr}$-activated resin was mixed with $2 \mathrm{ml}$ of protein $(1 \mathrm{mg} / \mathrm{ml})$ for $2 \mathrm{~h}$ at room temperature using an end-over-end mixer; next unreacted groups were blocked with Glycine $0.2 \mathrm{M} \mathrm{pH} 8$, for $2 \mathrm{~h}$ at room temperature and finally the resin was washed with phosphate buffered saline (PBS). Afterwards, $2 \mathrm{ml}$ of patient's serum were allowed to recirculate through the affinity column for $1 \mathrm{~h}$, in order for the specific Aabs to bind to their antigen. Washing steps were performed first with PBS, then with PBS-NaCl 0.5 M. Anti-Ro52 or an-
ti-Ro60 Aabs were eluted with Glycine $0.1 \mathrm{M} \mathrm{pH} 3.5$ and then $\mathrm{pH}$ 2.7, using an ÄKTA purifier system (GE Healthcare). Eluted fractions (1 ml each) were immediately neutralized with $1 \mathrm{M}$ Tris-HCl $\mathrm{pH} 8$ and subsequently tested for anti-Ro reactivity by a heterogeneous non-competitive ELISA, coated with the recombinant proteins, as described before [24]. High reactivity fractions were pooled (one pool for fractions eluted at $\mathrm{pH} 3.5$ and another for 2.7) and dialyzed against PBS. Pooled fractions were titrated by ELISA and their protein concentration determined by BCA Protein Assay Kit (Pierce, Rockford, IL, USA) using the extended protocol suggested by the manufacturer, in order to increase the sensitivity of the detection. Finally they were stored at $-80^{\circ} \mathrm{C}$ in glycerol $10 \%$ until the flow cytometric analysis began.

Reactivity against native Ro proteins was also evaluated for the pooled fractions, using an indirect immunofluorescence technique with commercially available Hep-2 cells (Kallestad, BIORAD, Houston, TX, USA), according to the manufacturers' instructions.

\subsection{In Vitro Model}

A human keratinocyte cell line, HaCaT, was kindly provided by Dr N. E. Fusenig (German Cancer Research Center, Heidelberg, Germany) [35]. HaCaT cells were cultured in plastic dishes and subconfluent cells were irradiated with $50 \mathrm{~mJ} / \mathrm{cm}^{2}$ of UVB light as previously described $[36,37]$ in order to obtain a high percentage of apoptotic cells. At least one dish was not exposed to UVB irradiation, but was handled in the same fashion as the irradiated cells, that is to say the culture medium was replaced by PBS before the irradiation. After irradiation, the PBS was immediately replaced with DMEM. All detached and attached cells were harvested and collected $24 \mathrm{~h}$ after UVB irradiation and they were incubated with PBS-total rabbit serum $1 / 10$, for $2 \mathrm{~h}$ at $4^{\circ} \mathrm{C}$ before the labeling for flow cytometry, to block Fc receptors in order to prevent unspecific binding of Igs.

Aabs binding to cell surface was measured by flow cytometry, incubating total irradiated cells $\left(1 \times 10^{6}\right.$ cells $)$ with patients' sera or affinity-purified anti-Ro52 or antiRo60 Aabs for $2 \mathrm{~h}$ at $4^{\circ} \mathrm{C}$, followed by rapid fixation with p-formaldehyde $2 \%$. Using this method, binding to cell surface is allowed and subsequent internalization of Aabs is prevented. Sera were used at a dilution of 1:100 in PBS-1\% BSA and purified Aabs were assayed undiluted. This was done in order to make the anti-Ro reactivity equivalent both in sera and purified Aabs for every patient. NHS (1:100), human purified IgG (hIgG, 0.1 $\mathrm{mg} / \mathrm{ml}$ ) and secondary antibody alone (UBC-unspecific binding control) were used as controls. Sera and NHS IgG masses used were equal, hIgG control was used in a 
concentration equivalent to one of the purified Aabs fraction which presented the highest mass. After washing twice with cold PBS, cells were incubated with secondary antibody FITC-conjugated goat anti-human IgG H + L (Bethyl, Montgomery, TX, USA) at a dilution of 1:100 in PBS-1\% BSA, for $2 \mathrm{~h}$ at room temperature. After the final washing with PBS cells were analyzed with a PAS-III PARTEC flow cytometer (Münster, Germany). In each experiment, the same first antibody labeling (sera, purified Aabs, NHS and hIgG) was also performed on non-irradiated cells.

\subsection{In Vivo Model}

In vivo assays were performed using male and female Crl: SKH-1-hrBR Hairless mice of 8 weeks of age, purchased from Charles River Laboratories. Animals were housed in quarters with 12/12 h light/dark cycle and maintained with water and food ad libitum.

Mice were divided into 4 groups of 10 animals, and a conventional intraperitoneal immunization schedule was performed with $100 \mu \mathrm{g}$ of each protein in complete Freund's adyuvant (CFA). Immunizations (total of 6) were made in 20-day intervals and sample taking (total of 3 ) in 40-day intervals. Groups were immunized with recombinant human Ro52 (Group 1), recombinant human Ro60 (Group 2), recombinant human Ro52 and Ro60 (Group 3), and the vehicle buffer in CFA (Group 4). Blood samples were taken by facial vein puncture and tested for reactivity against recombinant human Ro proteins by ELISA, as described before [24], and also against mice spleen extract in order to test autoreactivity [38].

When the animals' anti-Ro autoreactivity values became different from the control (group 4) in a statistically significant way, each group was divided in two, one group was irradiated (5 mice) and the other was mockedirradiated (5 mice). They were irradiated on their back with one minimal erythema dose $\left(200 \mathrm{~mJ} / \mathrm{cm}^{2}\right)$ of UVB, as described before [36]. After irradiation, animals were kept in the same conditions for $24 \mathrm{~h}$ and they were subsequently sacrificed. Non-irradiated mice were used as control and they were handled in the same fashion as the irradiated animals. Mice were sacrificed using a $\mathrm{CO}_{2}$ gas chamber, then dorsal skin tissue was obtained, one part was used for histological analysis and the rest was used to prepare epidermal homogenates.

In order to prepare epidermal homogenates total dorsal skin was immersed $30 \mathrm{~s}$ in water at $60{ }^{\circ} \mathrm{C}$, then the epidermis was scraped from the dermis using a blade and homogenized in $2 \mathrm{ml}$ of PBS, with $0.5 \mathrm{mg} / \mathrm{ml}$ EDTA and $0.174 \mu \mathrm{g} / \mathrm{ml}$ PMSF (Sigma Chemical Co) in a glass homogenizer with Teflon pestle, then centrifuged at $4{ }^{\circ} \mathrm{C}$ for $15 \mathrm{~min}$ at 12,000 rpm. Afterwards, total protein concen- tration in the supernatant was measured with the BCA Protein Assay kit (Pierce), using bovine serum albumin (BSA) as standard. Finally, the epidermal extracts were stored at $-70^{\circ} \mathrm{C}$ until the assays were done.

Cytokine quantification was performed by ELISA. Mouse TNF- $\alpha$ (epidermis extracts) was quantified using a non-competitive ELISA assay (BD Biosciences, San José, CA, USA), values were expressed as $\mathrm{pg} / \mathrm{mg}$ of protein. All assays were repeated at least three times.

Skin samples for histological analysis were fixed with $4 \%$ neutral formalin, and embedded in white paraffin. Samples were cut in $3 \mu \mathrm{m}$ sections and stained with hematoxylin-eosin (H-E).

\subsection{Data and Statistical Analysis}

Flow cytometric data was analyzed using Cyflogic software (version 1.2.1, CyFlo ltd, Turku, Finland). Graphical and statistical analyses were performed with GraphPad Prism 5.0 (GraphPad Software Inc., San Diego, CA, USA). For the in vitro model Analysis of Variance (ANOVA) followed by a Dunnet postest was performed to make multiple comparisons against a control value and a Student's t-test to compare means of two groups. For the in vivo model ANOVA followed by a Dunnet postest was performed in case of homoscedasticity and KruskalWallis (non parametric ANOVA) followed by a Dunn postest in case of heteroscedasticity. Results are expressed as mean $\pm \mathrm{SD}$; asterisks indicate significant at ${ }^{*} \mathrm{p}$ $<0.05,{ }^{* *} \mathrm{p}<0.01,{ }^{* * *} \mathrm{p}<0.001$.

\section{Results}

\subsection{Patients}

The study group consisted of 10 patients selected from a larger previous study; Table 1 shows their clinical characteristics and laboratory findings. There were 4 patients with CP (2 SLE and 2 sSS, and 6 patients without CP (1 SLE, 2 pSS and 3 sSS). Sjögren's Syndrome was found secondary to SLE or RA. All patients had anti-Ro52 and anti-Ro60 appropriate titers; except one (p4) which did not present reactivity for Ro60. The average disease evolution among all 10 patients was of 5 years and their median age was 48.5 years old (between 24 and 83 years old), with $90 \%$ being female.

\subsection{Affinity Purification of Aabs against Recombinant Ro/SS-A 52 and Ro/SS-A 60 $\mathrm{kDa}$}

Anti-Ro52 Aabs were purified from $2 \mathrm{ml}$ of serum using an affinity column with Ro52 protein coupled and anti-Ro60 Aabs were purified from another $2 \mathrm{ml}$ of serum using an affinity column with Ro60 coupled. Subsequent 
Table 1. Clinical and laboratory features of the 10 patients under study. SAP: systemic autoimmune pathology, CP: cutaneous photosensitivity. Purified Aabs titer correspond to $\mathrm{pH} 3.5$ pool, which was used for subsequent studies.

\begin{tabular}{|c|c|c|c|c|c|c|}
\hline \multirow[b]{3}{*}{ Patient num } & \multirow[b]{3}{*}{ SAP } & \multirow[b]{3}{*}{$\mathrm{CP}$} & \multicolumn{4}{|c|}{ Titer (log dil) } \\
\hline & & & \multicolumn{2}{|c|}{ Serum } & \multicolumn{2}{|c|}{ Purified Aabs } \\
\hline & & & anti-Ro52 & anti-Ro60 & anti-Ro52 & anti-Ro60 \\
\hline 1 & SLE & yes & 4.41 & 3.20 & 2.51 & 1.51 \\
\hline 2 & SLE & yes & 4.11 & 3.81 & 2.20 & 2.11 \\
\hline 3 & sSS (SLE) & yes & 2.30 & 2.60 & 1.00 & 1.20 \\
\hline 4 & sSS (SLE) & yes & 3.20 & - & 1.90 & - \\
\hline 5 & sSS (SLE) & no & 3.81 & 4.11 & 2.51 & 2.20 \\
\hline 6 & sSS (RA) & no & 5.61 & 2.90 & 4.01 & 1.81 \\
\hline 7 & sSS (RA) & no & 4.71 & 4.41 & 3.71 & 2.71 \\
\hline 8 & pSS & no & 4.41 & 3.81 & 2.81 & 2.11 \\
\hline 9 & pSS & no & 4.00 & 3.51 & 2.30 & 1.90 \\
\hline 10 & SLE & no & 4.11 & 3.20 & 2.20 & 1.81 \\
\hline
\end{tabular}

figures show patient num 6 (p6) as an illustrative example.

Figure 1(a) shows the purification profile of $\mathrm{p} 6$ serum, where the reactivity by ELISA of each eluted fraction can be seen. The efficiency of the purification procedure was evaluated by titration of the serum by ELISA, before and after the affinity-purification procedure. Figure 1(b) shows the titration sigmoidal curves and titers of p6 serum, which needed to be re-purified in order to obtain the majority of the anti-Ro52 Aabs present in the serum, but purified only once for anti-Ro60 Aabs. Several sera needed a second purification procedure; and high reactivity fractions from both chromatographies of each serum were afterwards pooled. The criteria used to decide if a serum sample did not need to be re-purified was reaching a titer $\leq 2.3$ (1/200 dilution) or a decrease in the titer $\geq 4$ dilutions with respects to the original sample.

For each patient, one pool was made from high reactivity fractions eluted at $\mathrm{pH} 3.5$ and another pool from the ones at $\mathrm{pH}$ 2.7. Figure 1(c) shows the corresponding titers of the pooled fractions, none of them presented cross-reactivity against the other protein (e.g. anti-Ro52 $\mathrm{pH} 3.5$ pool did not exhibit reactivity against Ro60Data not shown). Dialyzed pooled pH 3.5 fractions lost some anti-Ro reactivity with respects to the corresponding serum, but all of them retained enough reactivity in order to be used afterwards. Dialyzed pooled $\mathrm{pH} 2.7$ fractions lost almost all of their anti-Ro reactivity, so these fractions were not used afterwards. This reactivity decrease could be due to damage of the Aabs because of both low $\mathrm{pH}$ and manipulation, and also to sample dilu- tion. Titers of all sera and the corresponding purified Aabs (pH 3.5 pool) can be observed in Table 1. It is worth noticing that $\mathrm{p} 6$ is a special case for anti-Ro52, it has a significant high titer of those Aabs and it is the only patient whose pooled dialyzed $\mathrm{pH} 2.7$ fractions retained an adequate reactivity, as it can be seen in Figure 1(c). For all the other 9 patients the situation is similar to what happens with dialyzed $\mathrm{pH} 2.7$ fractions of anti-Ro60 from $\mathrm{p} 6$.

To confirm that purified Aabs were able to recognize the native antigen present in the cells, samples were tested by indirect immunofluorescence on Hep-2 cells. This is shown in Figure 2 for $\mathrm{p} 6$ as representative of the whole cohort, where it can be seen that the three samples were able to bind to the cells, presenting the typical speckled anti-Ro pattern. This was tested for all patients' sera and their corresponding purified Aabs, confirming their ability to recognize the native antigen. Controls (NHS, hIgG and UBC) are also shown, with no positive images, as expected.

\subsection{In Vitro Model}

In a previous study on HaCaT cells we have determined that irradiation with $50 \mathrm{~mJ} / \mathrm{cm}^{2}$ of UVB and evaluation of the cells $24 \mathrm{~h}$ post irradiation are the best conditions which produce the highest percentage of apoptotic cells with the lowest amount of living or necrotic ones [37]. Aabs binding to apoptotic keratinocyte's cell surface was evaluated by flow cytometry for the serum of each patient and the corresponding purified anti-Ro52 and antiRo60 Aabs. Figure 3(a) shows histograms with the 
(a) anti-Ro52
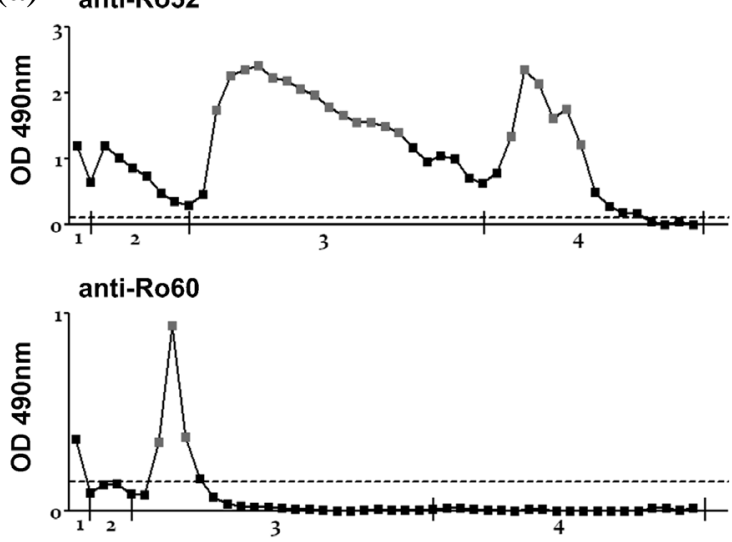

(b)
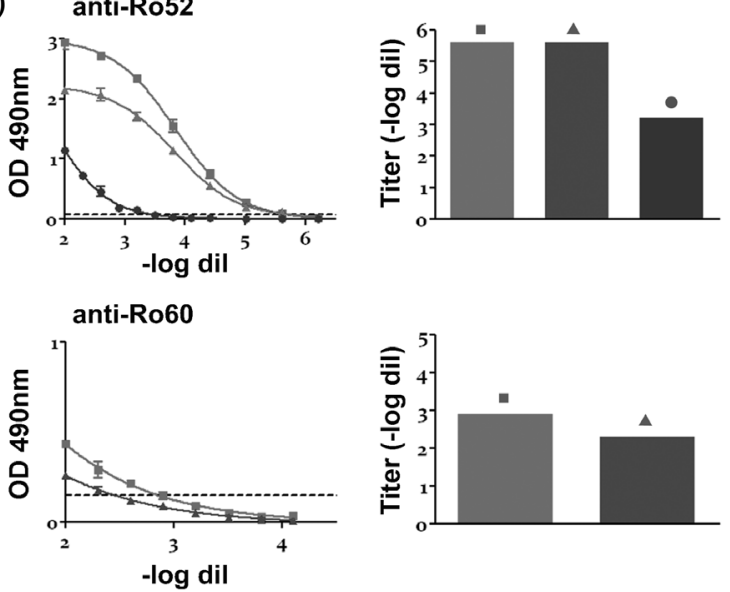

(c)

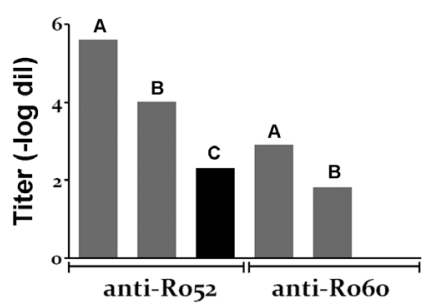

Figure 1. Affinity purification of autoantibodies (a) Profile of human anti-Ro52 and anti-Ro60 purified Aabs. Washing and elution, 1: PBS; 2: PBS-NaCl 0.5M; 3: Gly 0.1M pH 3.5; 4: Gly 0.1M pH 2.7. (b) Anti-Ro52 and anti-Ro60 serum titration (sigmoidal curves) and serum titers (column bar graph) before and after the affinity-purification procedure. I: before purification; $\Delta$ : after purification; $\bullet$ : after repurification (only for anti-Ro52). (c) Anti-Ro52 and anti-Ro60 titers of A: serum; B: purified Aabs (pH 3.5 pool); C: purified Aabs (pH 2.7 pool). In all graphs: reactivity determined by ELISA measuring OD at $490 \mathrm{~nm}$. Dotted line: cut off. All sera and purified Aabs from p6.

marker used to select the FITC-positive cells. As p6 serum was the one with the highest anti-Ro52 titer, and showed a very high fluorescence when incubated with irradiated cells (Irr), it was used in all experiments as a

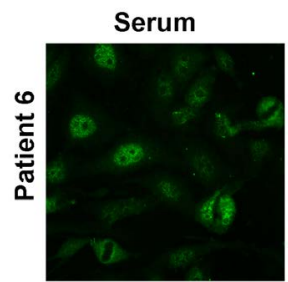

NHS
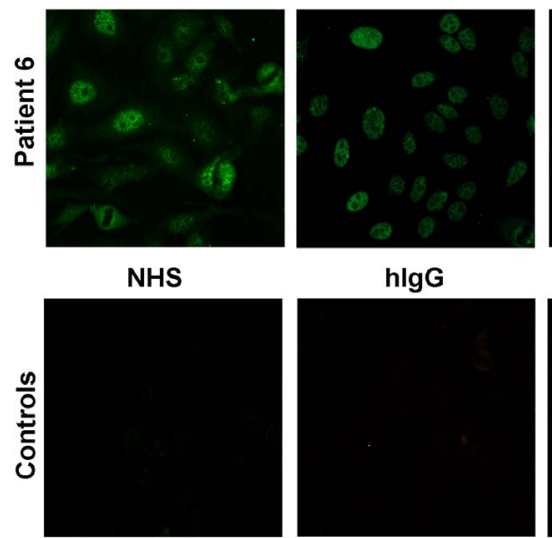

hlgG

Figure 2. Indirect immunofluorescence on Hep-2 cells. Sera and purified Aabs from p6 (anti-Ro52 and anti-Ro60) and controls (NHS, hIgG and UBC-unspecific binding control) are shown.

(a)

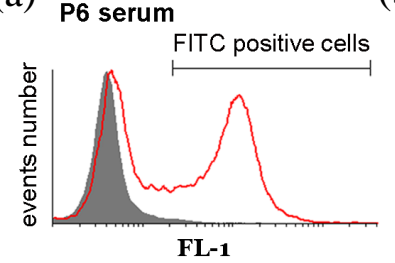

(b) NHS

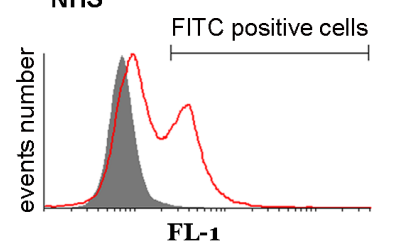

anti-Ro52

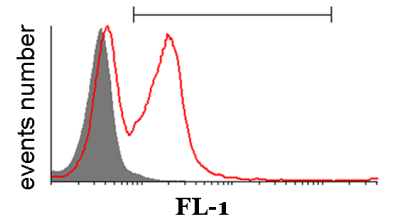

anti-Ro60
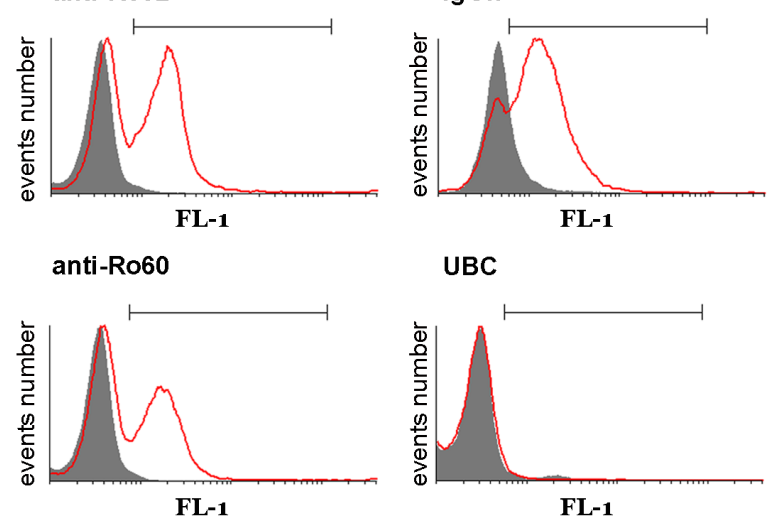

UBC

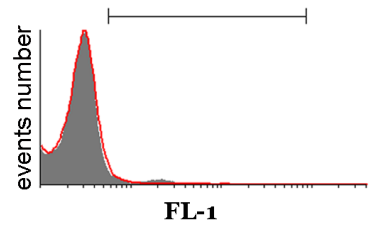

Figure 3. Aabs binding capacity to apoptotic keratinocyte's cell surface by flow cytometry. Histograms showing the number of events vs. FITC signal (FL1) correspond to (a) p6 samples: serum, purified anti-Ro52 Aabs and purified anti-Ro60 Aabs (b) controls: NHS, hIgG and UBC. Solid grey: samples on non-irradiated cells. Red: samples on irradiated cells. Marker: FITC positive cells.

positive control in order to set the FITC-positive cells marker and to standardize subsequent data processing. Figure 3(b) shows the histograms corresponding to controls: NHS, hIgG and UBC on irradiated as well as on non-irradiated cells (nIrr). Figure 4(a) shows the binding capacity to apoptotic keratinocyte's cell surface of all 10 
serum samples, expressed as Binding Index (BI) in arbitrary units. This BI was used to standardize variations between experiments and was calculated as follows; considering the mean fluorescence intensity (MFI) and the percentage of cells (\%) under the selection marker:

$$
\begin{gathered}
\text { Score }=(\% \times \text { MFI Irr cells })-(\% \times \text { MFI nIrr cells }) \\
\text { BI }=\frac{\text { Score patient } X}{\text { Score P6 }}
\end{gathered}
$$

Figures 4(b) and (c) show the BI of anti-Ro52 purified Aabs and anti-Ro60 respectively, for the 10 patients under study. BI for control NHS and hIgG were included in the graphs as a cut off; they were calculated as the mean of the BI of 5 independent experiments. These experiments prove that serum samples of the 10 patients did bind to keratinocytes' cell surface, showing BI which were statistically significant greater than the BI for control NHS. Their corresponding purified anti-Ro Aabs were also able to bind to the irradiated cells, both antiRo52 and anti-Ro60, most of them having a BI statistically significant different from the BI for control hIgG. When patients were grouped according to the presence or absence of CP (Figure 4, lower row), there were no differences found in the mean BI from the two groups.

\subsection{In Vivo Model}

Immunized SKH-1 Hairless mice were tested for serum reactivity against recombinant human Ro proteins and also against mice spleen extract in order to test autoreactivity, by ELISA. By the end of the immunization schedule all animals from groups 1,2 and 3 were able to react against both types of proteins (Figure 5), in a statistically significant different way compared to group 4 (control).

Mice with circulating anti-Ro Aabs were divided in two groups, one was irradiated and the other mockedirradiated (on day 144 of the immunization schedule). They were sacrificed $24 \mathrm{~h}$ later and histological analysis and cytokine quantification were performed. Figure 6 shows the skin sections of one representative mouse from each group of animals, where it can be seen that there were no differences between immunized non irradiated groups and the corresponding irradiated ones, except for the known effects of UVB irradiation on the skin [36,39]. Only one group showed a slight difference with respects to the others: when irradiated, group 1 presented a slight inflammatory infiltrate not seen in the other groups. TNF- $\alpha$ production quantified in epidermal extracts can be seen in Figure 7. It is observed that TNF- $\alpha$ is barely not detectable in non irradiated mice, while its levels increased in all irradiated animals as expected, but showing no statistically significant differences between groups.

\section{Discussion}

In the present study a group of 10 Argentinean middle
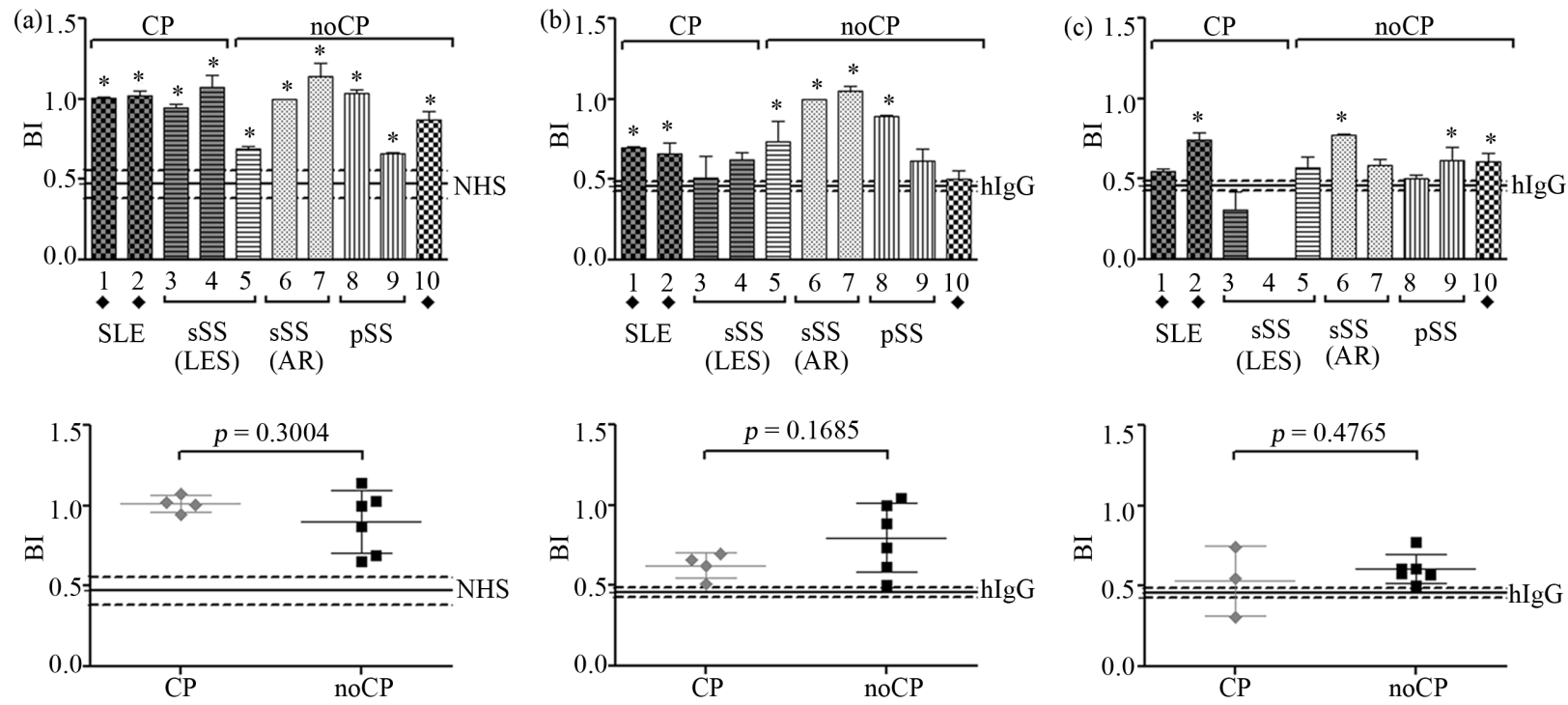

Figure 4. Binding Index (BI) to apoptotic keratinocyte's cell surface of all ten patients, expressed as arbitrary units. (a) Serum samples (b) Anti-Ro52 purified Aabs (c) Anti-Ro60 purified Aabs. BI for control NHS and hIgG were included as cut off (mean \pm 2 SD). Patients were grouped according to the presence (CP) or absence of $C P($ noCP) and their diagnosed disease is also shown. Upper Row: BI for each patient and each of their corresponding samples. Samples statistically different from control $(p<0.05)$ are indicated with an asterisk ( $\left(^{*}\right)$, ANOVA followed by a Dunnet postest was used. Lower Row: mean BI for each patient. The exact $p$ value is indicated, obtained by a Student's t-test. 
(a)

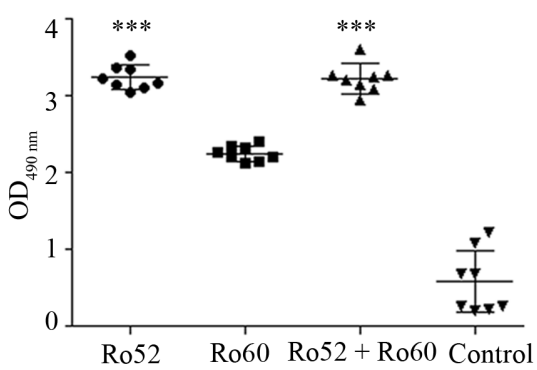

(b)

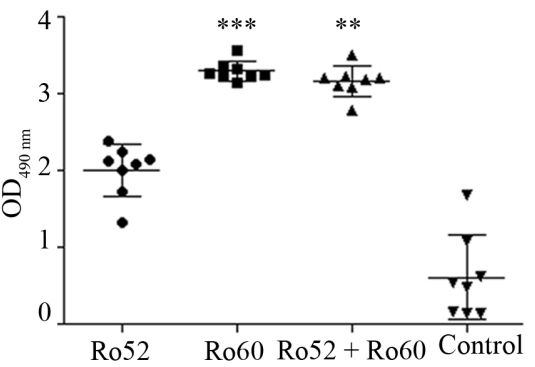

(c)

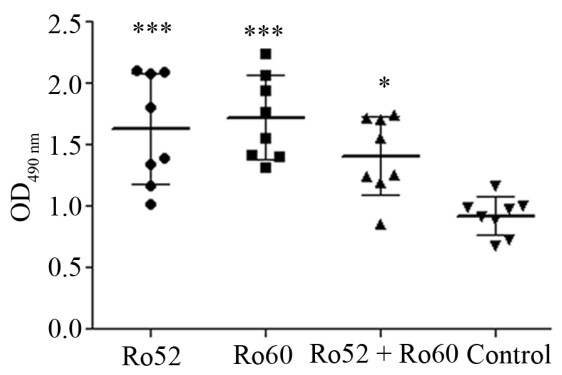

Figure 5. Mice serum reactivity by ELISA at day 120 of the immunization schedule. The three groups of immunized mice were tested against (a) Recombinant human Ro52 (b) Recombinant human Ro60 (c) Mice spleen extract. ANOVA or Kruskal-Wallis were performed, and samples statistically different from control group are indicated with an asterisk ( ${ }^{*} p<$ $\left.0.05,{ }^{* *} \mathrm{p}<0.01,{ }^{* * *} \mathrm{p}<0.001\right)$.
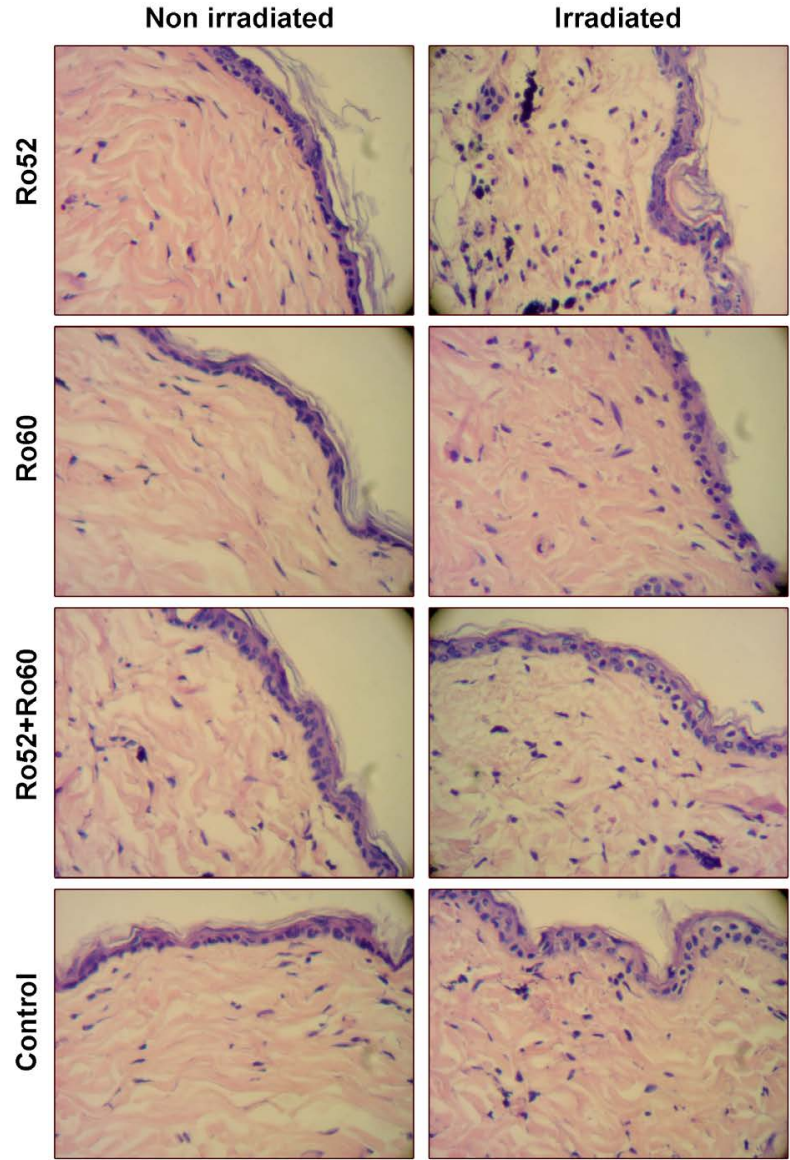

Figure 6. H-E skin sections of one representative mouse from each group of animals. Left: immunized (or control) non irradiated groups. Right: immunized (or control) irradiated groups.

aged patients was studied. They were selected from a cohort of 169 individuals, attempting to compose two homogeneous groups: one group consisting of patients with CP and the other one of patients without CP. All of them with high anti-Ro52 and/or anti-Ro60 titers, and

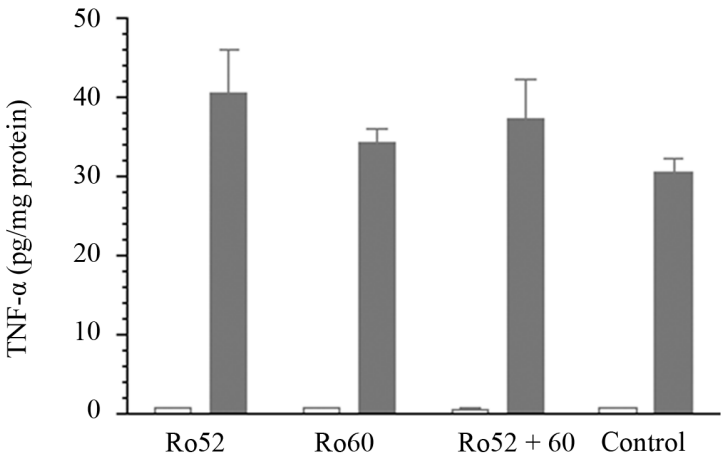

Figure 7. TNF- $\alpha$ quantification in epidermal extracts. White bars: immunized (or control) non irradiated groups. Grey bars: immunized (or control) irradiated groups. KruskalWallis (non parametric ANOVA) followed by a Dunn posttest was performed, differences between immunized irradiated groups were not significant.

with the same kind of SAP: SLE or SS. Purification of anti-Ro52 and anti-Ro60 individually from each patient's serum was feasible, some of them needed to be re-purified, and all the Aabs pure pooled fractions exhibited reactivity against recombinant and also native antigens, and a titer which was adequate to use in subsequent experiments and suitable to make comparisons with the corresponding serum.

HaCaT cells were irradiated with $50 \mathrm{~mJ} / \mathrm{cm}^{2}$ of UVB light and labeled for flow cytometry $24 \mathrm{~h}$ after, in this way high levels of apoptotic cells were obtained, as it has been previously described by us [37]. Therefore, UVB irradiated cells are a good in vitro model to test binding of Aabs to cell surfaced relocalized autoantigens. Binding capacity to apoptotic keratinocyte's cell surface was expressed as BI to standardize the results, and NHS and hIgG were used as control of basal normal binding, and they were set as a binding cut off. The entire set of sera and Aabs were tested on non-irradiated cells, exhibiting no binding to these living cells. When cells were UVB- 
irradiated they underwent apoptosis, relocalizing the autoantigens, therefore sera of the 10 patients were able to bind to the keratinocyte's cell surface, showing greater BI values than NHS. NHS and hIgG on irradiated cells showed higher fluorescence intensity compared to values on non-irradiated cells, this could be explained since it has been proven that Ro52 autoantigen is capable of binding human IgG1 nonspecifically through its heavy chain $[40,41]$. It can be concluded that serum of the 10 patients did bind to apoptotic keratinocytes, as well as their corresponding purified anti-Ro Aabs. All anti-Ro52 and anti-Ro60 purified Aabs but one show BI values greater than control ones, though due to a difference in the IgG mass between purified Aabs and control hIgG some values did not reach statistical significance. It can also be seen that there is no relationship between the sera binding capacity and the presence or absence of CP, not either with the SAPs. We are aware that 10 patients is not a significantly elevated number, nevertheless, we believe that the conclusions obtained are legitimate, not only because they are coherent among themselves, but also because they are in agreement with other studies.

These results are in agreement with our recent study on 169 patients' sera [24] of the direct relationship between the occurrence of CP and the presence of certain Aabs. We found that there was no association between the presence of anti-Ro Aabs and the occurrence of CP. This was true for anti-Ro52 as well as for anti-Ro60, both for presence and titer. Besides our results, there are many other reports where no direct relationship was found between clinical photosensitivity and the presence of anti-Ro Aabs, such as studies in Japanese, black South African SLE patients and others [22,42,43]. Taking into account that anti-Ro Aabs are not a sine qua non of photosensitivity because 1) they are not commonly found in association with DLE, though DLE patients do present CP 2) healthy people can manifest CP without anti-Ro Aabs 3 ) there is the above-mentioned case of pSS patients which do have high titer of anti-Ro circulating Aabs, but do not show signs of $\mathrm{CP}$, it is reasonable to think that anti-Ro Aabs are not necessarily the causative or the only responsible of causing CP.

However, there are many studies that indicate a positive relationship between anti-Ro Aabs and CP, like the ones which measure the binding of sera containing antiRo Aabs from patients to self-derived irradiated keratinocytes [18-20]. These studies were always performed with sera which were tested by various techniques to be only specific for one autoantigen, among 4 or 5 different ones. The usage of this kind of monoespecific sera has many disadvantages, like 1) not being completely representative of a SAP's patient 2) having the monoespecificity tested against only a few well-known autoantigens and 3) the usage of serum instead of pure Aabs, implying that other antibodies (not tested in the "monoespecificity assay") could be present in those monoespecific sera and interacting with the irradiated keratinocytes. Those assays were also performed on patients' own keratinocytes, and it has been shown that keratinocytes from cutaneous LE patients showed a higher susceptibility to a singledose UVB light irradiation compared to keratinocytes from normal controls, and that the binding of Aabs was more up-regulated when cultured keratinocytes were reacted with autologous sera [29]. Anyhow, experiments were always performed using sera and we suggest that, according to our results, there might be other Aabs implicated in the pathogenesis of CP that are not essentially anti-Ro Aabs, and that those Aabs could be the anti-Sm ones, due to the fact that we-and also other authors$[24,27,43]$ have previously found a statistically significant direct positive association between $\mathrm{CP}$ and high reactivity anti-Sm Aabs.

We have used an in vivo model to also test the relationship between anti-Ro Aabs and CP. Since there is similarity between the sequence of the human and mice Ro proteins [44,45], and also there is spreading of mice immune response towards an autoimmune profile [46,47], then we succeed in generating Aabs against murine Ro52 and/or Ro60 autoantigens in the immunized mice. Mice with circulating anti-Ro Aabs were divided in 2 groups, one was irradiated and the other mocked-irradiated. TNF- $\alpha$ production showed no statistical differences between the 3 immunized-irradiated groups and the non immunized-irradiated control group; neither did the evaluated parameters by histological analysis, except for the slight inflammatory infiltrate found in the irradiated group immunized with Ro52. This fact suggests that the single presence of anti-Ro Aabs is not the only requirement for the development of $\mathrm{CP}$, and many other mediators might be involved in the pathogenesis of the manifestation.

\section{Conclusion}

To sum up, using the two approaches studied in the present work, it can be concluded that anti-Ro Aabs are not related to the CP phenomenon, or at least, they are not the only responsible for the CP pathogenesis. These results agree with some studies on the role of the Aabs in $\mathrm{CP}$, considering anti-Ro Aabs not as the only ones involved in the manifestation; and disagrees with many other authors, who believe in the strong association between these two events. Nonetheless, many issues remain to be investigated and solved before any definite conclusion can be drawn about the pathogenesis of photosensitivity. 


\section{Acknowledgements}

The authors would like to thank Dr. María Pino and Gustavo Nasswetter MD for their collaboration in the diagnosis of the patients. We would also like to thank the IDEHU Animal Technicians and Dr Daniela Ureta for their excellent technical assistance with animal care and with the flow cytometry analysis, respectively.

This study was funded by grants from the University of Buenos Aires (UBACyT B099 and B023) and by the National Research Council (CONICET PIP 5837 and 02299). MLP and EC are supported by a fellowship from CONICET, AF DHGM and JL are members of the CONICET Research Career Program, and ADF is supported by a fellowship from the UBA. The funders had no role in study design, data collection and analysis, decision to publish, or preparation of the manuscript.

\section{REFERENCES}

[1] A. J. Wysenbeek, D. A. Block and J. F. Fries, "Prevalence and Expression of Photosensitivity in Systemic Lupus Erythematosus,” Annals of the Rheumatic Diseases, Vol. 48, No. 6, 1989, pp. 461-463. http://dx.doi.org/10.1136/ard.48.6.461

[2] N. Scheinfeld and V. A. Deleo, "Photosensitivity in Lupus Erythematosus," Photodermatology, Photoimmunology \& Photomedicine, Vol. 20, No. 5, 2004, pp. 272-279. http://dx.doi.org/10.1111/j.1600-0781.2004.00094.x

[3] M. C. Hochberg, "Updating the American College of Rheumatology Revised Criteria for the Classification of Systemic Lupus Erythematosus," Arthritis \& Rheumatology, Vol. 40, No. 9, 1997, p. 1725. http://dx.doi.org/10.1002/art.1780400928

[4] J. Wenzel, S. Zahn and T. Tuting, "Pathogenesis of $\mathrm{Cu}-$ taneous Lupus Erythematosus: Common and Different Features in Distinct Subsets,” Lupus, Vol. 19, No. 9, 2010, pp. 1020-1028. http://dx.doi.org/10.1177/0961203310370046

[5] R. D. Sontheimer, "Photoimmunology of Lupus Erythematosus and Dermatomyositis: A Speculative Review," Photochemistry and Photobiology, Vol. 63, No. 5, 1996, pp. 583-594. http://dx.doi.org/10.1111/j.1751-1097.1996.tb05660.x

[6] T. P. Millard, J. L. Hawk and J. M. McGregor, "Photosensitivity in Lupus,” Lupus, Vol. 9, No. 1, 2000, pp. 310. http://dx.doi.org/10.1177/096120330000900103

[7] F. Furukawa, "Photosensitivity in Cutaneous Lupus Erythematosus: Lessons from Mice and Men," Journal of Dermatological Science, Vol. 33, No. 2, 2003, pp. 81-89. http://dx.doi.org/10.1016/j.jdermsci.2003.08.005

[8] G. Fabini, S. A. Rutjes, C. Zimmermann, G. J. Pruijn and G. Steiner, "Analysis of the Molecular Composition of Ro Ribonucleoprotein Complexes. Identification of Novel Y RNA-Binding Proteins,” European Journal of Biochemistry, Vol. 267, No. 9, 2000, pp. 2778-2789. http://dx.doi.org/10.1046/j.1432-1327.2000.01298.x
[9] M. Herrmann, R. E. Voll and J. R. Kalden, "Etiopathogenesis of Systemic Lupus Erythematosus,” Immunology Today, Vol. 21, No. 9, 2000, pp. 424-426. http://dx.doi.org/10.1016/S0167-5699(00)01675-3

[10] M. Reichlin and R. Scofield, "SS-A (Ro) Autoantibodies," In: J. Peter and Y. Shoenfeld, Eds., Autoantibodies, Elsevier, Amsterdam, 1996, pp. 783-788. http://dx.doi.org/10.1016/B978-044482383-0/50120-5

[11] C. A. von Muhlen and E. M. Tan, "Autoantibodies in the Diagnosis of Systemic Rheumatic Diseases," Seminars in Arthritis and Rheumatism, Vol. 24, No. 5, 1995, pp. 323358. http://dx.doi.org/10.1016/S0049-0172(95)80004-2

[12] S. E. Sonesson, S. Salomonsson, L. A. Jacobsson, K. Bremme and M. Wahren-Herlenius, "Signs of First-Degree Heart Block Occur in One-Third of Fetuses of Pregnant Women with Anti-SSA/Ro 52-kd Antibodies," Arthritis \& Rheumatology, Vol. 50, No. 4, 2004, pp. 12531261. http://dx.doi.org/10.1002/art.20126

[13] K. Tsuzaka, T. Fujii, M. Akizuki, T. Mimori, T. Tojo, et al., "Clinical Significance of Antibodies to Native or Denatured 60-kd or 52-kd Ro/SS-A Proteins in Sjogren's Syndrome,” Arthritis \& Rheumatology, Vol. 37, No. 1, 1994, pp. 88-92. http://dx.doi.org/10.1371/journal.pone.0009599

[14] L. D. Heinlen, M. T. McClain, L. L. Ritterhouse, B. F. Bruner, C. C. Edgerton, et al., "60 kD Ro and nRNP A Frequently Initiate Human Lupus Autoimmunity,” PLoS One, Vol. 5, No. 3, 2010, p. e9599. http://dx.doi.org/10.1371/journal.pone.0009599

[15] L. Casciola-Rosen, G. Anhalt and A. Rosen, "Autoantigens Targeted in Systemic Lupus Erythematosus Are Clustered in Two Populations of Surface Structures on Apoptotic Keratinocytes,” Journal of Experimental Medicine, Vol. 179, No. 4, 1994, pp. 1317-1330. http://dx.doi.org/10.1084/jem.179.4.1317

[16] K. Ayukawa, S. Taniguchi, J. Masumoto, S. Hashimoto, H. Sarvotham, et al., "La Autoantigen Is Cleaved in the $\mathrm{COOH}$ Terminus and Loses the Nuclear Localization Signal during Apoptosis,” Journal of Biological Chemistry, Vol. 275, No. 44, 2000, pp. 34465-34470. http://dx.doi.org/10.1074/jbc.M003673200

[17] D. Mevorach, J. L. Zhou, X. Song and K. B. Elkon, "Systemic Exposure to Irradiated Apoptotic Cells Induces Autoantibody Production,” Journal of Experimental Medicine, Vol. 188, No. 2, 1998, pp. 387-392. http://dx.doi.org/10.1084/jem.188.2.387

[18] F. Furukawa, T. Itoh, H. Wakita, H. Yagi, Y. Tokura, et al., "Keratinocytes from Patients with Lupus Erythematosus Show Enhanced Cytotoxicity to Ultraviolet Radiation and to Antibody-Mediated Cytotoxicity," Clinical \& Experimental Immunology, Vol. 118, No. 1, 1999, pp. 164-170. http://dx.doi.org/10.1046/j.1365-2249.1999.01026.x

[19] F. Furukawa, M. Kashihara-Sawami, M. B. Lyons and D. A. Norris, "Binding of Antibodies to the Extractable Nuclear Antigens SS-A/Ro and SS-B/La Is Induced on the Surface of Human Keratinocytes by Ultraviolet Light (UVL): Implications for the Pathogenesis of Photosensi- 
tive Cutaneous Lupus,” Journal of Investigative Dermatology, Vol. 94, No. 1, 1990, pp. 77-85. http://dx.doi.org/10.1111/1523-1747.ep12873930

[20] T. D. Golan, K. B. Elkon, A. E. Gharavi and J. G. Krueger, "Enhanced Membrane Binding of Autoantibodies to Cultured Keratinocytes of Systemic Lupus Erythematosus Patients after Ultraviolet B/Ultraviolet A Irradiation," Journal of Clinical Investigation, Vol. 90, No. 3, 1992, pp. 1067-1076. http://dx.doi.org/10.1172/JCI115922

[21] F. Furukawa, “Antinuclear Antibody-Keratinocyte Interactions in Photosensitive Cutaneous Lupus Erythematosus,” Histology and Histopathology, Vol. 14, No. 2, 1999, pp. 627-633.

[22] P. G. Sutej, A. J. Gear, R. C. Morrison, M. Tikly, M. de Beer, et al., "Photosensitivity and Anti-Ro (SS-A) Antibodies in Black Patients with Systemic Lupus Erythematosus (SLE)," British Journal of Rheumatology, Vol. 28, No. 4, 1989, pp. 321-324. http://dx.doi.org/10.1046/j.1365-2133.2003.05379.x

[23] C. J. Sanders, H. Van Weelden, G. A. Kazzaz, V. Sigurdsson, J. Toonstra, et al., "Photosensitivity in Patients with Lupus Erythematosus: A Clinical and Photobiological Study of 100 Patients Using a Prolonged Phototest Protocol,” British Journal of Dermatology, Vol. 149, No. 1, 2003, pp. 131-137. http://dx.doi.org/10.1046/j.1365-2133.2003.05379.x

[24] M. L. Paz, D. H. Gonzalez Maglio, M. Pino, A. Ferrari, F. S. Weill, et al., "Anti-Ribonucleoproteins Autoantibodies in Patients with Systemic Autoimmune Diseases. Relation with Cutaneous Photosensitivity," Clinical Rheumatology, Vol. 30, No. 2, 2011, pp. 209-216. http://dx.doi.org/10.1007/s10067-010-1481-8

[25] K. Yamamoto, "Pathogenesis of Sjogren's Syndrome," Autoimmunity Reviews, Vol. 2, No. 1, 2003, pp. 13-18. http://dx.doi.org/10.1016/S1568-9972(02)00121-0

[26] N. Nardi, P. Brito-Zeron, M. Ramos-Casals, S. Aguilo, R. Cervera, et al., "Circulating Auto-Antibodies against Nuclear and Non-Nuclear Antigens in Primary Sjogren's Syndrome: Prevalence and Clinical Significance in 335 Patients," Clinical Rheumatology, Vol. 25, No. 3, 2006, pp. 341-346. http://dx.doi.org/10.1007/s10067-005-0059-3

[27] A. Doria, C. Biasinutto, A. Ghirardello, E. Sartori, R. Rondinone, et al., "Photosensitivity in Systemic Lupus Erythematosus: Laboratory Testing of ARA/ACR Definition,” Lupus, Vol. 5, No. 4, 1996, pp. 263-268. http://dx.doi.org/10.1177/096120339600500404

[28] D. Ioannides, B. D. Golden, J. P. Buyon ans J. C. Bystryn, "Expression of SS-A/Ro and SS-B/La Antigens in Skin Biopsy Specimens of Patients with Photosensitive Forms of Lupus Erythematosus,” Archives of Dermatology, Vol. 136, No. 3, 2000, pp. 340-346. http://dx.doi.org/10.1001/archderm.136.3.340

[29] F. Furukawa, H. Kanauchi and S. Imamura, "Susceptibility to UVB Light in Cultured Keratinocytes of Cutaneous Lupus Erythematosus,” Dermatology, Vol. 189, Suppl. 1, 1994, pp. 18-23. http://dx.doi.org/10.1159/000246922

[30] L. A. Lee, K. K. Gaither, S. N. Coulter, D. A. Norris and
J. B. Harley, "Pattern of Cutaneous Immunoglobulin G Deposition in Subacute Cutaneous Lupus Erythematosus Is Reproduced by Infusing Purified Anti-Ro (SSA) Autoantibodies into Human Skin-Grafted Mice,” Journal of Clinical Investigation, Vol. 83, No. 5, 1989, pp. 15561562. http://dx.doi.org/10.1172/JCI114052

[31] T. L. Davis, C. B. Lyde, B. M. Davis and R. D. Sontheimer, "Perturbation of Experimental Ultraviolet light-Induced Erythema by Passive Transfer of Serum from Subacute Cutaneous Lupus Erythematosus Patients,” Journal of Investigative Dermatology, Vol. 92, No. 4, 1989, pp. 573-577. http://dx.doi.org/10.1111/1523-1747.ep12709604

[32] I. Maczynska, B. Millo, V. Ratajczak-Stefanska, R. Maleszka, Z. Szych, et al., "Proinflammatory Cytokine (IL1beta, IL-6, IL-12, IL-18 and TNF-Alpha) Levels in Sera of Patients with Subacute Cutaneous Lupus Erythematosus (SCLE)," Immunology Letters, Vol. 102, No. 1, 2006, pp. 79-82. http://dx.doi.org/10.1016/j.imlet.2005.08.001

[33] C. H. Orteu, R. D. Sontheimer and J. P. Dutz, “The Pathophysiology of Photosensitivity in Lupus Erythematosus,” Photodermatology, Photoimmunology \& Photomedicine, Vol. 17, No. 3, 2001, pp. 95-113. http://dx.doi.org/10.1034/j.1600-0781.2001.170301.x

[34] S. Louhimies, "Directive 86/609/EEC on the Protection of Animals Used for Experimental and Other Scientific Purposes," Alternatives to Laboratory Animals, Vol. 30, Suppl. 2, 2002, pp. 217-219.

[35] P. Boukamp, R. T. Petrussevska, D. Breitkreutz, J. Hornung, A. Markham, et al., "Normal Keratinization in a Spontaneously Immortalized Aneuploid Human Keratinocyte Cell Line,” Journal of Cell Biology, Vol. 106, No. 3, 1988, pp. 761-771. http://dx.doi.org/10.1083/jcb.106.3.761

[36] M. L. Paz, A. Ferrari, F. S. Weill, J. Leoni and D. H. Maglio, "Time-Course Evaluation and Treatment of Skin Inflammatory Immune Response after Ultraviolet B Irradiation,” Cytokine, Vol. 44, No. 1, 2008, pp. 70-77. http://dx.doi.org/10.1016/j.cyto.2008.06.012

[37] M. L. Paz, D. H. Gonzalez Maglio, F. S. Weill, J. Bustamante and J. Leoni, "Mitochondrial Dysfunction and Cellular Stress Progression after Ultraviolet B Irradiation in Human Keratinocytes," Photodermatology, Photoimmunology \& Photomedicine, Vol. 24, No. 3, 2008, pp. $115-122$ http://dx.doi.org/10.1111/j.1600-0781.2008.00348.x

[38] M. Reichlin, M. Rader and J. B. Harley, “Autoimmune Response to the Ro/SSA Particle Is Directed to the Human Antigen," Clinical \& Experimental Immunology, Vol. 76, No. 3, 1989, pp. 373-377.

[39] D. H. Gonzalez Maglio, M. L. Paz, A. Ferrari, F. S. Weill, A. Czerniczyniec, et al., "Skin Damage and Mitochondrial Dysfunction after Acute Ultraviolet B Irradiation: Relationship with Nitric Oxide Production," Photodermatology, Photoimmunology \& Photomedicine, Vol. 21, No. 6, 2005, pp. 311-317. http://dx.doi.org/10.1111/j.1600-0781.2005.00185.x

[40] Y. S. Yang, M. C. Yang, B. Wang and J. C. Weissler, 
“Autoantigen Ro52 Directly Interacts with Human IgG Heavy Chain in Vivo in Mammalian Cells," Molecular Immunology, Vol. 37, No. 10, 2000, pp. 591-602. http://dx.doi.org/10.1016/S0161-5890(00)00068-7

[41] M. Takahata, M. Bohgaki, T. Tsukiyama, T. Kondo, M. Asaka, et al., "Ro52 Functionally Interacts with IgG1 and Regulates Its Quality Control via the ERAD System," Molecular Immunology, Vol. 45, No. 7, 2008, pp. 20452054. http://dx.doi.org/10.1016/j.molimm.2007.10.023

[42] F. Furukawa and M. Muto, "Ethnic Differences in Immunogenetic Features and Photosensitivity of Cutaneous Lupus Erythematosus," Archives of Dermatological Research, Vol. 301, No. 1, 2009, pp. 111-115. http://dx.doi.org/10.1007/s00403-008-0897-3

[43] K. H. Ching, P. D. Burbelo, C. Tipton, C. Wei, M. Petri, et al., "Two Major Autoantibody Clusters in Systemic Lupus Erythematosus,” PLoS One, Vol. 7, No. 2, 2012, Article ID: e32001. http://dx.doi.org/10.1371/journal.pone.0032001

[44] C. L. Keech, T. P. Gordon and J. McCluskey, "Structural Differences between the Human and Mouse 52-kD Ro
Autoantigens Associated with Poorly Conserved Autoantibody Activity across Species,” Clinical \& Experimental Immunology, Vol. 104, No. 2, 1996, pp. 255-263.

[45] M. Wahren, K. Skarstein, I. Blange, I. Pettersson and R. Jonsson, "MRL/lpr Mice Produce Anti-Ro 52,000 MW Antibodies: Detection, Analysis of Specificity and Site of Production,” Immunology, Vol. 83, No. 1, 1994, pp. 9-15.

[46] U. S. Deshmukh, J. E. Lewis, F. Gaskin, C. C. Kannapell, S. T. Waters, et al., "Immune Responses to Ro60 and Its Peptides in Mice. I. The Nature of the Immunogen and Endogenous Autoantigen Determine the Specificities of the Induced Autoantibodies," Journal of Experimental Medicine, Vol. 189, No. 3, 1999, pp. 531-540. http://dx.doi.org/10.1084/jem.189.3.531

[47] F. Topfer, T. Gordon and J. McCluskey, "Intra- and Intermolecular Spreading of Autoimmunity Involving the Nuclear Self-Antigens La (SS-B) and Ro (SS-A)," Proceedings of the National Academy of Sciences of the United States of America, Vol. 92, No. 3, 1995, pp. 875879. http://dx.doi.org/10.1073/pnas.92.3.875 